POS $\quad \begin{aligned} & \text { PROCEEDINGS } \\ & \text { OF SCIENCE }\end{aligned}$

\title{
Preface
}

\section{Carleton DeTar}

Department of Physics, University of Utah, Salt Lake City, UT 84112, USA

E-mail: detar@physics.utah.edu

\section{Colin Morningstar}

Department of Physics, Carnegie Mellon University, Pittsburgh, PA 15213, USA

E-mail: colin_morningstar@emu.edu

\section{David Richards}

Thomas Jefferson National Accelerator Facility, Newport News, VA 23606, USA

E-mail: dgrejlab.org

XXIVth International Symposium on Lattice Field Theory

July 23-28, 2006

Tucson, Arizona, USA 


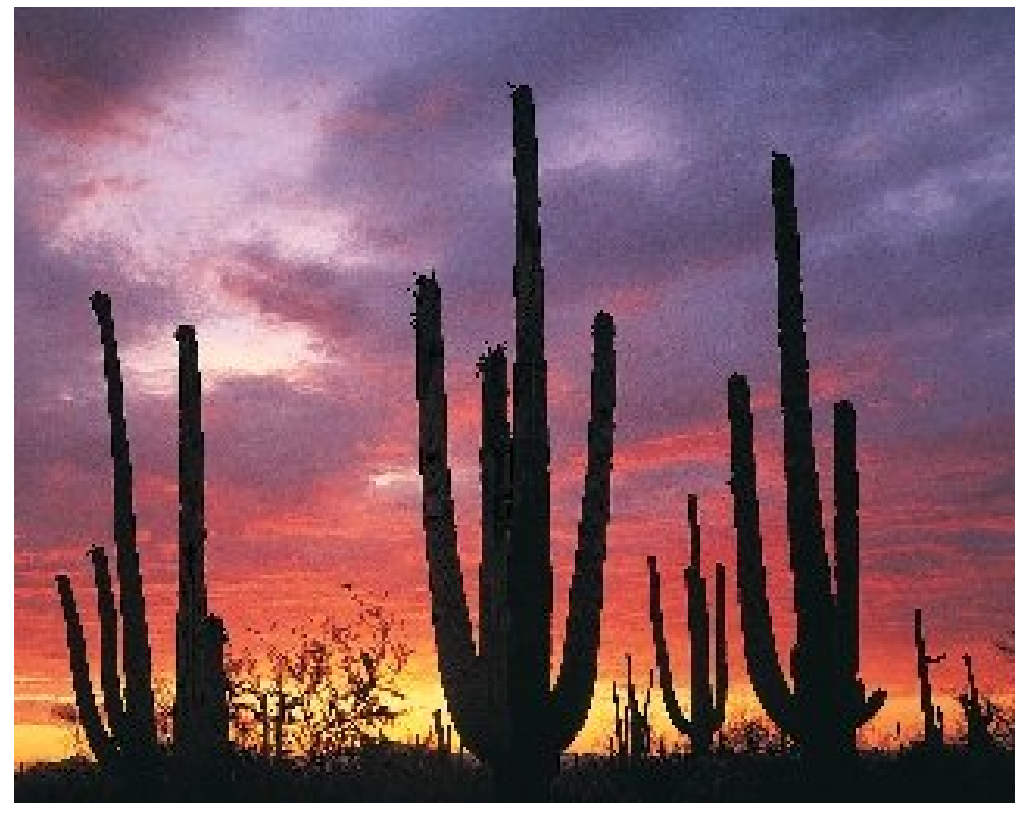

Lattice 2006, the XXIVth International Symposium on Lattice Field Theory, was held from July 23-28, 2006 at the Starr Pass Hotel near Tucson, Arizona, USA, hosted by the University of Arizona Physics Department. About 240 participants from many countries attended the symposium. The scientific program, formed by the Program Committee with guidance from the International Advisory Committee, contained 25 plenary session talks and 193 parallel session contributions (talks and posters). Most of these are recorded in this proceedings.

Topics in lattice QCD included: hadron spectroscopy; hadronic interactions and structure; algorithms, machines, and networks; chiral symmetry; QCD confinement and topology; quark masses, gauge couplings, and renormalization; electroweak decays and mixing; high temperature and density; and theoretical developments. Topics beyond QCD included large Nc, Higgs, SUSY, gravity, and strings. The keynote talk, "QCD physics beyond the standard model and LHC searches," was given by Ann Nelson.

Participants enjoyed the facilities of the Starr Pass Hotel, where conference sessions were held, as well as lodging and most of the meals. The first conference excursion went to Old Tucson, once a studio where western movies were made, for an evening of entertainment and a (partly cloudy) star party. The second excursion was to the Arizona Sonoran Desert Museum where participants learned about desert animal and plant life and enjoyed the conference banquet.

The following organizations provided support that helped make this an enjoyable and rewarding conference. The organizers would like to gratefully acknowledge: Brookhaven National Laboratory, the U.S. Department of Energy, Fermi National Accelerator Lab, Intel Corporation, Jefferson Lab, the Metropolitan Tucson Convention and Visitors Bureau, The National Science Foundation, Office Depot, the Raytheon Corporation, the Research Corporation, the Stanford Linear Accelerator Center, the Tucson Amateur Astronomy Association, the University of Arizona, and the University of Arizona Physics Department.

Before, during, and after the conference, the conference staff kept everything running smoothly: Don Cooney, Kavan Cooney, Mike Eklund, Ana Favela, Marlene Hughes, Ron Landis, Adriana 
Peabody, Galina Pecherskaya, Ali Renner, Dru Renner, Alex Santa Cruz, David Sarich, Donna Swibold, Phillip Toussaint, Roberta Toussaint, Rachael Volner, Karen Woerner, and Mary Young.

The members of the Program Committee were

$\begin{array}{ll}\text { Tom Blum } & \text { University of Connecticut } \\ \text { Mike Creutz } & \text { Brookhaven National Lab } \\ \text { Carleton DeTar } & \text { University of Utah } \\ \text { Frithjof Karsch } & \text { Brookhaven National Lab } \\ \text { Andreas Kronfeld } & \text { Fermilab } \\ \text { Colin Morningstar } & \text { Carnegie Mellon University } \\ \text { Dave Richards } & \text { Jefferson Lab } \\ \text { Junko Shigemitsu } & \text { The Ohio University } \\ \text { Doug Toussaint } & \text { University of Arizona }\end{array}$

The members of the International Advisory Committee were

$\begin{array}{ll}\text { Sinya Aoki } & \text { Tsukuba University } \\ \text { Claude Bernard } & \text { Washington University } \\ \text { Jonathan Flynn } & \text { Southampton University } \\ \text { Philippe de Forcrand } & \text { ETH Zurich } \\ \text { Rajiv Gavai } & \text { TIFR Mumbai } \\ \text { Anna Hasenfratz } & \text { University of Colorado } \\ \text { Shoji Hashimoto } & \text { KEK Tsukuba } \\ \text { Julius Kuti } & \text { University of California, San Diego } \\ \text { Maria Lombardo } & \text { INFN Frascati } \\ \text { Herbert Neuberger } & \text { Rutgers University } \\ \text { Michael Peardon } & \text { Trinity College, Dublin } \\ \text { Andreas Schaefer } & \text { University of Regensburg } \\ \text { Howard Trottier } & \text { Simon Fraser University } \\ \text { Peter Weisz } & \text { MPI Munich } \\ \text { Tilo Wettig } & \text { University of Regensburg } \\ \text { Anthony Williams } & \text { University of Adelaide }\end{array}$




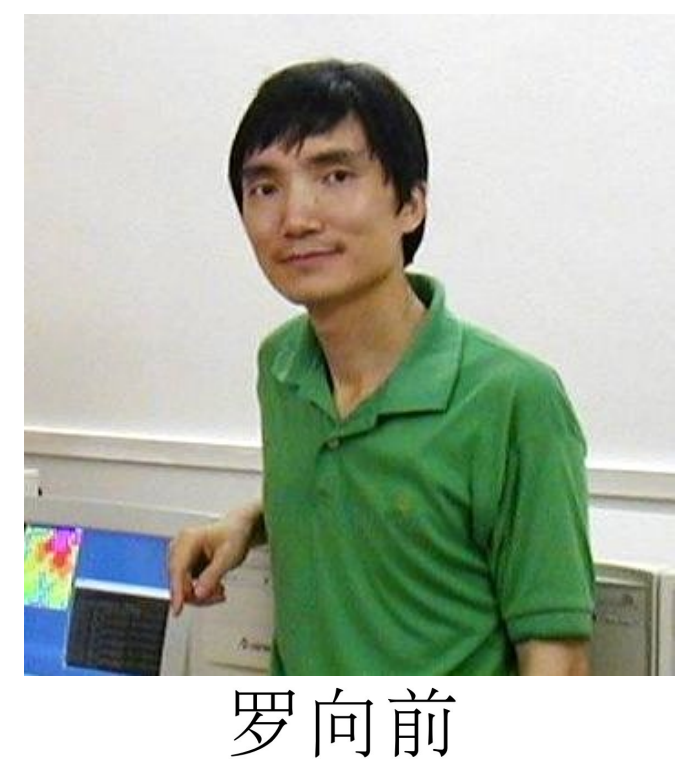

Xiang-Qian Luo 1961-2006

This volume is dedicated to the memory of Xiang-Qian Luo, who died from cancer on October 23, 2006 in Guangzhou, China.

Xiang-Qian was born on November 11, 1961 in Guangzhou to Chun-Hua Luo and Jin-Xiao Luo during a time of economic difficulty in China. Believing that the future must surely hold brighter prospects, they named him "Xiang-Qian", meaning "Forward".

In 1978 he sat for the first national Chinese university entrance exam and gained admittance into the prestigious Tianjin University. There he studied precision instruments, receiving his bachelors degree in July 1982. After graduating, Xiang-Qian developed an interest in physics and in 1985 he began graduate physics studies at Zhongshan University in Guangzhou. He completed his Master of Science and PhD under Shou-Hong Guo in 1988 and 1991, respectively.

In 1990 and 1991 Xiang-Qian was a visiting scholar at the University of California, Santa Barbara and Fermilab in the USA. From 1991 to 1993 Xiang-Qian was a postdoc at the University of Zargoza in Spain, and from 1993 to 1995 he received a joint postdoctoral appointment in Germany between DESY and the German Supercomputer Center in Jülich. Finally, in 1995 he returned to his hometown and became an Associate Professor of theoretical physics at Zhongshan University. He became a full professor in 1999. Xiang-Qian was also a regular visiting professor at Laval University in Quebec.

At Zhongshan University Xiang-Qian championed lattice field theory in China. He collaborated with physicists around the world and hosted two international workshops at Zhongshan University focusing on lattice field theory. In his time at Zhongshan University he supervised six PhD students, eight Masters students, and three postdocs. Xiang-Qian was an author on more than 140 physics publications including over 75 journal articles.

In his career, Xiang-Qian Luo was indeed influential in moving forward lattice field theory, as well as the careers of many young physicists. He is remembered as a good friend and colleague by physicists around the world. Xiang-Qian Luo is survived by his parents, his wife Danya $\mathrm{Xu}$, and his son Simon. 